\title{
Home Automation System with Universally Used Mobile Application Platform
}

\author{
Deepti Shastri (Assistant professor) \\ Department of electronics and communication engg, Shri Venkateshwara University, \\ NH-24, Rajabpur, Gajraula Distt: Amroha (India)
}

\begin{abstract}
This paper describes an investigation into the potential for different mobile application on home automation systems. It considers problems with their implementation, discusses possible solutions through various Mobile application platform and different network technologies and indicates how to optimize the use of such systems. The home is an eternal, heterogeneous, distributed computing environment which certainly requires a careful study before developing any suitable Home Automation System (HAS) that will accomplish its requirements. Now a days, mobile applications made were targeted to a particular Operating System only which restricted there usability. With the limitations of above in mind there is a need of a universal mobile application development platform. So that an application can be developed in a universal XML format which can be easily ported to any other mobile devices. Home automation system on one of the Operating System and creation of a unique XML document that can be placed over the server which can be adapted by any other mobile device without any platform issues. The XML format which controls the layout of the screen remains common, only the part which needs to be coded on every platform is the downloading of the XML file from the server and parsing it. This reduces a lot of coding effort as the design part is coded only once, and the same file can be used by every other platform. All the House hold activities are controlled by a smartphone which is connected to a server containing the XML file via an internet connection. We have taken Home Automation as one of our application, but the same principle can be implemented in any other applications like Mobile Themes, Games etc.
\end{abstract}

Keywords: Home network, Automation, iOS, Universal Mobile Application, XML.

\section{INTRODUCTION}

The rapid growth in mobile communication system in present era is changing people's life and work style. In present time mobiles are integrated handheld computers equipped with all communication capabilities These mobile phones allow users to download and run various applications. It is used to perform various operations with lot of mobile's application using various mobiles operating system. Thus we can say mobile is a real intelligent system. But even after immense work on mobile platform the compatibility factor is always a main constraint. The constraint is the Operating System of the mobile device. This makes the application to be used in a specified operating system. It also refers to the version of an operating system. The main objective of this paper is Universal Mobile Application Development which creates a platform for application portability \& flexibility between various mobile devices. Universal Mobile Application Development will run across virtually all mobile platforms without the limitation of operating system. By using Universal Mobile Application, we can resolve the problem of compatibility of operating system that was take place when we use any other operating system to implement home automation system. Home automation system that is used to perform and monitoring various the house hold activities and reduce the man power .It also reduce probability of errors because human beings are more prone to errors. The home appliances are controlled by the home server, which operates according to the user commands received from the mobile phone via internet. A key to the home automation system is the capability for remote operation. These systems rely on the internet as the medium for communication and generally feature friendly graphical user interfaces. The Internet-based approach requires a home server running on an Internet connected personal computer all the time. In our proposed system the home server is built upon a separate Server, which will have the repository of the XML files intended to the operating system and the version being used. It also takes into the account the different screen size and resolution functionalities allowing a user to control and monitor any variables related to the home by using any smart phone.

\section{DeSigning MeThodology}

The Universal Mobile Application Development methodology is achieved through application of the following principles:

The application functionality is delivered to the mobile device through an internet protocol thus enabling all web-enabled smartphones to universally access the application. Universal Mobile Application 
Development applications are hosted on an application server hence all processing for the mobile device is performed on the back-end server.

The client intended application provides maximum level of functionality and computing power. The mobile client application works in tandem with the interface bringing mobile-relevant functionality to the user. We are not only going to implement this in Android Operating system but also in Blackberry OS and if possible in Apple iOS too. With different Operating System running the same application, they will update the server with the latest status of the application. The architecture of the system looks like as shown in Figure 1.

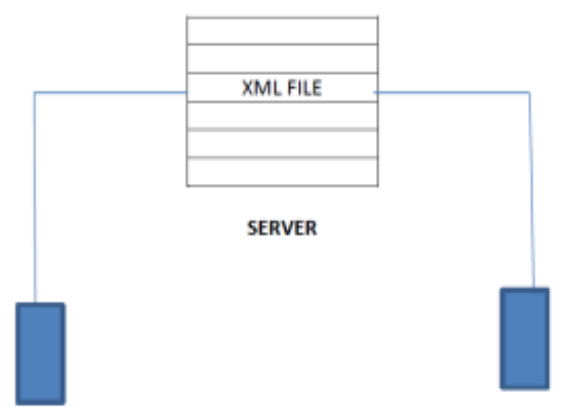

SMARTDEVICE 1

SMARTDEVICE 2

Figure: 1 Architecture of overall system with universal XML file placed on the server

\subsection{Application}

III. GRAPHICAL USER INTERFACE FOR MOBILE

The mobile phone is the interface between the user and the home automation system. It has two tasks:

1. Providing a user-friendly interface for the user to input control commands and view system's feedback.

2. Sending user commands to and receiving system's feedback from the home server.

An Android based Smartphone with enabled internet is chosen in our design due to the portability of Java and the fast increasing popularity of android-enabled mobiles. Another Operating system with which we are working is Blackberry. We will also try to work with the most advanced operating system designed by Apple Inc. iOS. The use of Blackberry makes work simple as the base is Java for developing purpose, so there is just a need of porting files from Android platform. The portability of Java ensures that applications developed in

Java languages are portable across different mobiles from different manufacturers. Since Android has a rich library of application program interfaces (API) providing functions such as graphical user interface, sending and receiving SMS messages and communicating via GPRS.Android applications for mobile phones can be easily developed by using Android development tools commonly known as ADT. In addition, phone manufactures provide their respective handset emulators for developers to test their applications on before testing on real phones. The major reason behind choosing Android, Blackberry and iOS is due to the market share they have achieved. The activity classification feature and synchronizing features make them outstanding in the field

of smartphones.

\subsection{Programming Cellular Phone}

The working of the home automation using Universal Mobile Application Development is as follows:-

1. A house will be fully home automated on a press of a button like locking doors, ON/OFF Tube lights and Bulbs, Fans, TV, AC etc.

2. The house members residing in this house may have different smartphones of different companies

3. Our application Universal Mobile Application Development will be installed on every smart phone device. These phones have to connect to the server for the first time to give the details of all the attributes of the particular smart phone.

4. After verification the $\mathrm{xml}$ file with its version number is downloaded on the Smartphone with the latest updates available. 
5. Now the house members can start using this application through which they can toggle the functions of all the home automation and can also get the latest update of the house where they can track the activities of other members.

6. Every change made is written back on the server xml, this ensures that the server gets updated instantly.

7. This feature will revolutionize the home automation industry as all functionality will be implemented on the server and the changes made will affect the xml file on server.

8. The client application present on the mobile will only have code to download and pa parse xml file on to sd card, and will return back the unique identification number of the components to the server.

9. The server will read the unique id and accordingly will make the changes in the server xml as all functionality is provided on the server side.

The flow of events that take place in the system is shown in Figure 2. This starts from server identifying the user providing it with the required XML file and allowing read/write operations within a session. As shown in Figure 3 , the applications will start with an authentication and a request of an appropriate IP address which will download the XML file once for initiating the software application. The IP address is given a token from the server which will identify all its properties and resources. This makes the client application with a proper session details and unique functionality intended to a specific user. The whole application is controlled by the XML file making in an effort of universal development of the same application in different Operating Systems.

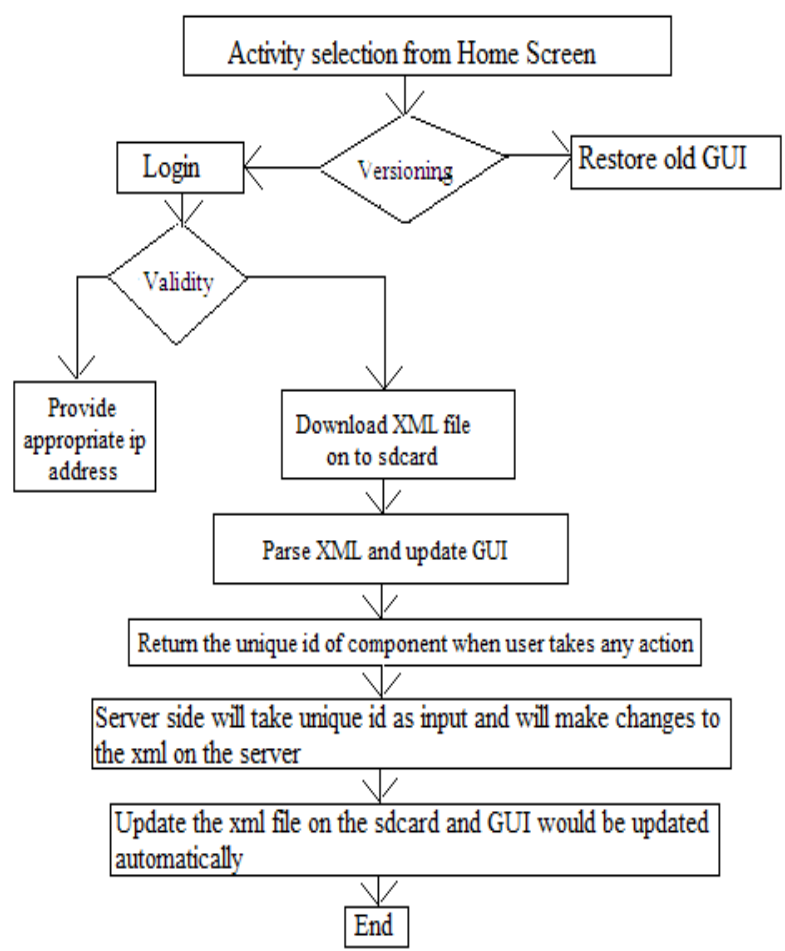

Figure: 3 Flow Chart

The file is downloaded from the server on to the SD card of the mobile device which makes the parsing of files faster and efficient. The application is divided in zones and its respective appliances. All these are shown in Figure 4 and Figure 5. As shown in Figure 6 application also performs dynamic updating of XML file on click events. We see that how the XML file is updated which makes the whole process simple and transparent. We have explicitly been using image views for a fantastic user experience. This particular feature of XML can be easily ported to different Operating Systems. All the images are loaded through a zip file and we can add views dynamically with keeping in mind the screen resolution of a mobile device. Now on the similar basis this will also work on the other platforms, the only change would be to program the download and parsing of file, which is already implemented. The graphical part will remain the same in the other platforms. 


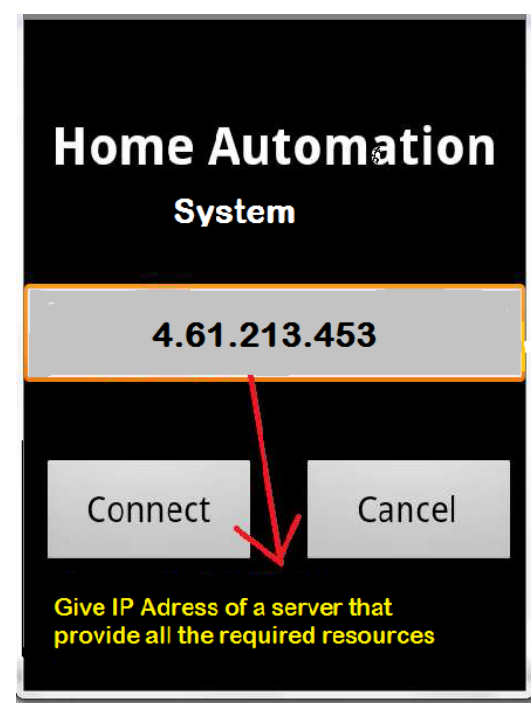

Figure: 3 Sign In Screen i.e.. Used To Connect With The Server

The file is downloaded from the server on to the sdcard of the mobile device which makes the parsing of files faster and efficient. The application is divided in zones and its respective appliances. All these are shown in Figure 4 and Figure 5. As shown in Figure 6 application also performs dynamic updating of XML file on click events. We see that how the XML file is updated which makes the whole process simple and transparent. We have explicitly been using image views for a fantastic user experience. This particular feature of XML can be easily ported to different Operating Systems. All the images are loaded through a zip file and we can add views dynamically with keeping in mind the screen resolution of a mobile device. Now on the similar basis this will also work on the other platforms, the only change would be to program the download and parsing of file, which is already implemented. The graphical part will remain the same in the other platforms.

\section{HARDWARE INTERFACE}

As for Home Automation application the hardware requirement is basically a Smartphone with a server placed as a backend. A hardware which will communicate with the home appliances will be placed inside the house which will listen to the instructions from server and accordingly will take necessary action. This hardware is nothing but the Hardware Modulator, which will have the function of reading the server commands and connecting to the hardware devices in response of which the devices will do the appropriate function.

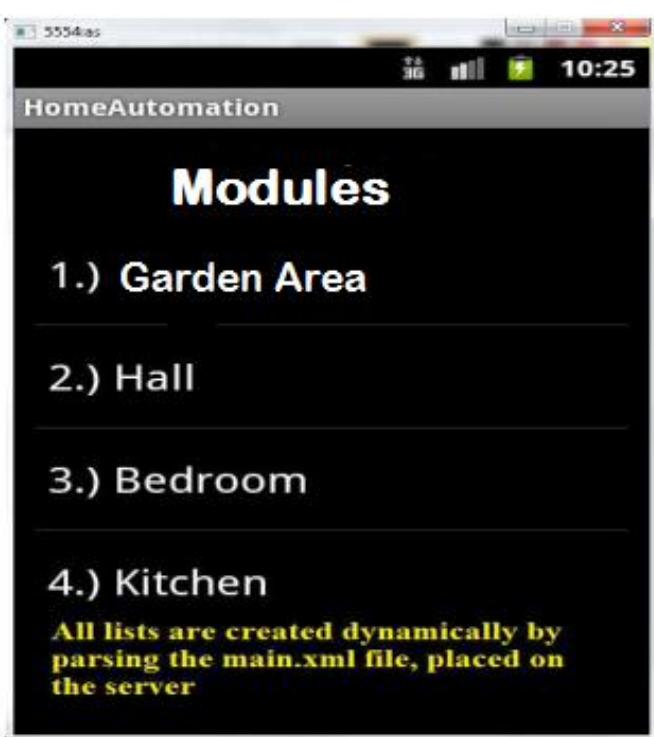

Figure: 4 List of various Modules connected with the server i.e. available on Sign in screen

For e.g.: If user wants to switch On the bulb then he will click the required button on the Smartphone and that will give a message to the Server which will command the Hardware Modulator to switch on the bulb. Our main 
focus is on the software part and which will provide the interfaces to execute these functions. The Hardware Modulator which is already present in the market will be just an extension as a connecting device. When it comes to hardware PRO-100 from Zigbee is the most efficient as it provides all the hardware interfaces to the software through its protocols

\section{USER INTERFACE}

The system interacts with the user with the help of a smart phone. When the user switches on the application, it first tries to connect to server and get the details of GUI from it. If the connection is not available then it restores the most recently used page as its default page placed in the SD card. According to ADA (American Disability Act) it is mandatory that it should work out for even disabled person, so in the case of a blind person operating a Smartphone should possess an audio interaction which can help the person to communicate in an easy way.

\section{ADVANTAGES OF UNIVERSAL MOBILE APPLICATION DEVELOPMENT}

1. Cross-platform mobile frameworks being open source has support from a large community of developers.

2. Increases sales/profits.

3. Augments your brand like never before.

4. Users can have access the apps from various places instead of one.

5. Once written, used everywhere coding of application, It saves lot of development time.

6. Distribute same app to a huge user base.

7. Achieve more diversity on different app development platforms.

8. Get option of integrating native features of smartphones/mobiles.

9. Problem of versioning system is removed as both GUI and functionality code for all the components of the XML file is provided on the server side.

10. This ensures that if an update is available then the old version need not be removed to use the new version. User can now buy application once and use it on any mobile or web platform that supports it.

\section{FUTURE WORK}

The work on Android is done and it is also feasible on Blackberry OS as it becomes easy to import the java files and the main universal part i.e. the XML will remain the same in the entire context. If this is possible on two platforms it opens the market to all the OS, as the crux is the transport of dynamism provided by the XML files. This makes it all platform compatible very soon in future. As the application we are developing is wholly internet based the server functionality can be put in cloud which makes sense as it gives faster access and saves the cost of maintaining the servers also. So Cloud Computing can be used for cost effectiveness and maintenance benefits.

\section{CONCLUSION}

The design and implementation of a novel mobile-based home automation system without Operating System constraint is presented. The design consists of a mobile phone with Android or Blackberry or iOS applications, a cellular modem, and a microcontroller (basically the Hardware). The home appliances are controlled by the microcontroller, which operates according to the user commands received from the mobile phone via the modem. Such a design transforms a mobile phone into a portable remote controller for home automation. It is noted that the proposed system is not restricted to home automation, it can be applied directly to remote control of many industrial devices.

\section{REFERENCES}

[1]. Chen, M. K. (1994). Practical design for computer speech recognition on PC. Flag Press Corp.

[2]. Franky Lam Nicole Lam Raymond K. Wong, "Performance evaluation of XSYNC: an efficient synchronizer for mobile xml data," iccs 2002, pp. 651-655, 6 Feb 2002.

[3]. Huang, C. J. (2003). Hierarchical grown blue-trees-An effective topology for Bluetooth scatter-nets. Master thesis, E.E. Department of National Chung-Sun University.

[4]. Le, H. P., Zayegh, A., \& Singh, J. (2003). A 12-bit high performance low cost pipeline ADC. Electronics, Circuits and Systems.

[5]. Lin, S.C. (2003). Communication protocol of message package of BT Net. Master thesis, Electronic Engineering Department of Fen-Kai University

[6]. Hsu, C. L., \& Chen, F. S. (2004). Theory and practical works of 8051/8951. Chuen-Hwa Technology Book Store.

[7]. Cheng, H. Y. (2005). Design and practical project of linking BT with microprocessor. Master thesis, E.E. department of National Cheng-Kung University.

[8]. Usman Ali, Syed Junaid Nawaz, Nazish Jawad, “A Real-time Control System for Home/Office appliances automation, from mobile device through GPRS network," IEEE Trans. Consumer Electron., pp. 854-857, 6 Feb 2006.

[9]. Jian-she Jin, Jing Jin+, Yong-hui Wang, Ke Zhao, Jia-jun Hu, "Development of Remote-Controlled Ray-Ti International Communication Ltd. Corp. (2007). Experiment data-book of BT EVAL LAB. 
[10]. Shi, Y. Y., Liu, J., \& Liu, R. S. (2001). Single-chip speech recognition system based on 8051 microcontroller core. Consumer Electronics, IEEE Transactions, 47(1), 149-153.

[11]. Tu, C. Y., Kuo, C. K., Lin, C. H., \& Lin, M. C. (2007). The microprogramming SPCE061 chips design. Chir-Kal Technology Ltd. Company.

[12]. Wang, S. C. (2007). Speech signals processing. Taipei: Chuan-hwa Bookstore

[13]. Chuang, C. B. (2008). Digital and analog IC Lab. Taipei: Chuan-hwa Bookstore.

[14]. Fan, I. C. (2009). Visual basic and RS232 serial communication port. Wen-Qwei Information Ltd. Corp

[15]. Home Automation System with Wireless Sensor Network," Fifth IEEE International Symposium on Embedded Computing, pp. 169-173, 2008.

[16]. Mahmoud shaker Nasr,Fahtha H. A.salem Azwai, "Friendly Home Automation System Using Cell Phone and J2ME with Feedback Instant Voice Messages," IEEE Trans. Consumer Electron, pp. 531- 538, 8 Nov. 2009.

[17]. K. Atukorala, D. Wijekoon, M. Tharugasini, I. Perera, C. Silva, "SmartEye - Integrated solution to home automation, security and monitoring through mobile phones," 2009 Third International Conference on Next Generation Mobile Applications, Services and Technologies, pp. 64-69, 2009. 